\title{
Diversity of Carbonyl Compounds in Biogas and Natural Gas Revealed Using High-Resolution Mass Spectrometry and Non-Target Analysis
}

\author{
Christopher P. Alaimo, Yin Li, Peter G. Green, \\ Michael J. Kleeman, and Thomas M. Young* \\ Department of Civil \& Environmental Engineering \\ University of California, One Shields Ave., Davis, CA 95616
}

\section{List of Supporting Information Tables}

(Tables can be found in the supporting information Excel file)

Table S1. LC-qTOF Parameters

Table S2. MS-DIAL Parameters

Table S3. Raw MS-DIAL Output

Table S4. Filtered Data

\section{List of Supporting Information Figures}

Figure S1. Flow chart representing the data analysis workflow, from sample analysis on the LC-QTOFMS to the final data set.

Figure S2. The Schymanski scale; confidence levels for the identification of non-target features in HRMS data. Taken from "Identifying Small Molecules via High Resolution Mass Spectrometry: Communicating Confidence" by Schymanski et. al. (2014). ${ }^{19}$

Figure S3. LC-qTOF chromatogram of a derivatized 2,5-hexanedione standard. From left to right: unreacted 2,4-DNPH, the two stereoisomers of the singly-derivatized compound, and the three stereoisomers of the doubly-derivatized compound.

Figure S4. Extracted masses consistent with singly and doubly-derivatized hexanedione isomers extracted from a food waste digester biogas sample (bottom). Note that the retention times differ from those in the derivatized standard because the sample likely contains different structural isomers of hexanedione. 


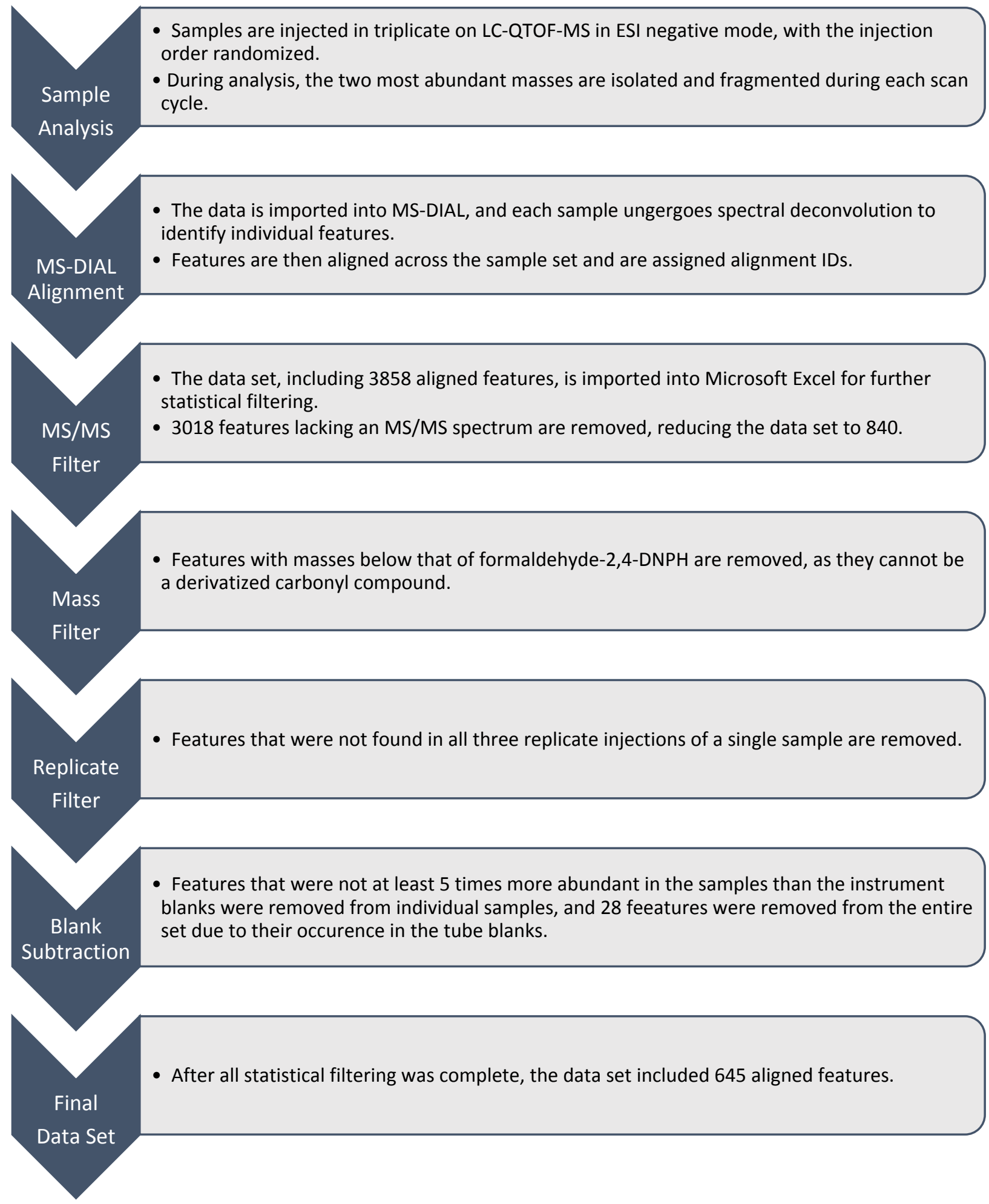

Figure S1. Flow chart representing the data analysis workflow, from sample analysis on the LC-QTOFMS to the final data set. 


\begin{tabular}{|c|l|}
\hline Confidence & \multicolumn{1}{c|}{ Criteria } \\
\hline Level 1 & $\begin{array}{l}\text { The compound has been confirmed through retention time and mass spectral } \\
\text { comparison to a reference analytical standard. }\end{array}$ \\
\hline Level 3 & $\begin{array}{l}\text { The feature has an unambiguous molecular formula that includes integer multiples of } \\
\text { the DNPH moiety, with a corresponding underivatized formula that is able to form a } \\
\text { plausible carbonyl compound. }\end{array}$ \\
\hline Level 5 & $\begin{array}{l}\text { The feature has an exact mass, but based on the formula assignment criteria no } \\
\text { formula could be assigned that meets the threshold for level 3. }\end{array}$ \\
\hline
\end{tabular}

Figure S2. Identification confidence levels for the non-target features in this study, along with their criteria. Based on the Schymanski scale found in "Identifying Small Molecules via High Resolution Mass Spectrometry: Communicating Confidence" by Schymanski et. al. (2014). ${ }^{19}$

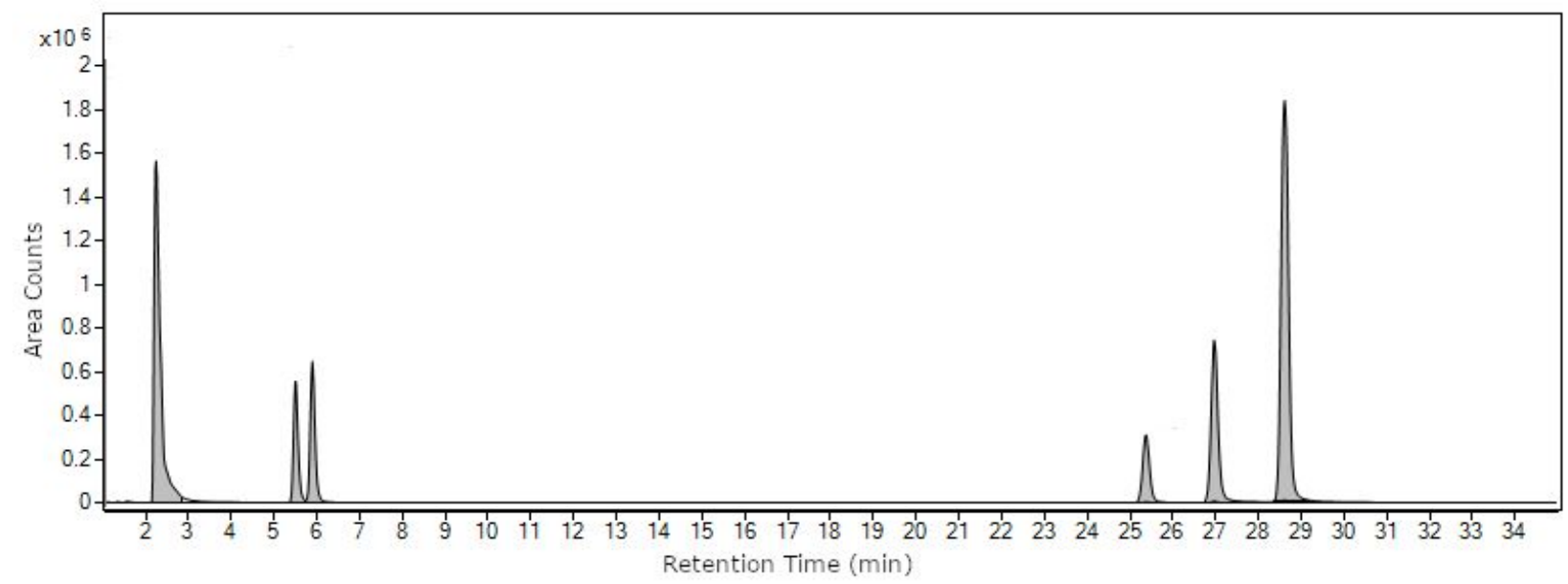

Figure S3. LC-qTOF chromatogram of a derivatized 2,5-hexanedione standard. From left to right: unreacted 2,4-DNPH, the two stereoisomers of the singly-derivatized compound, and the three stereoisomers of the doubly-derivatized compound. 


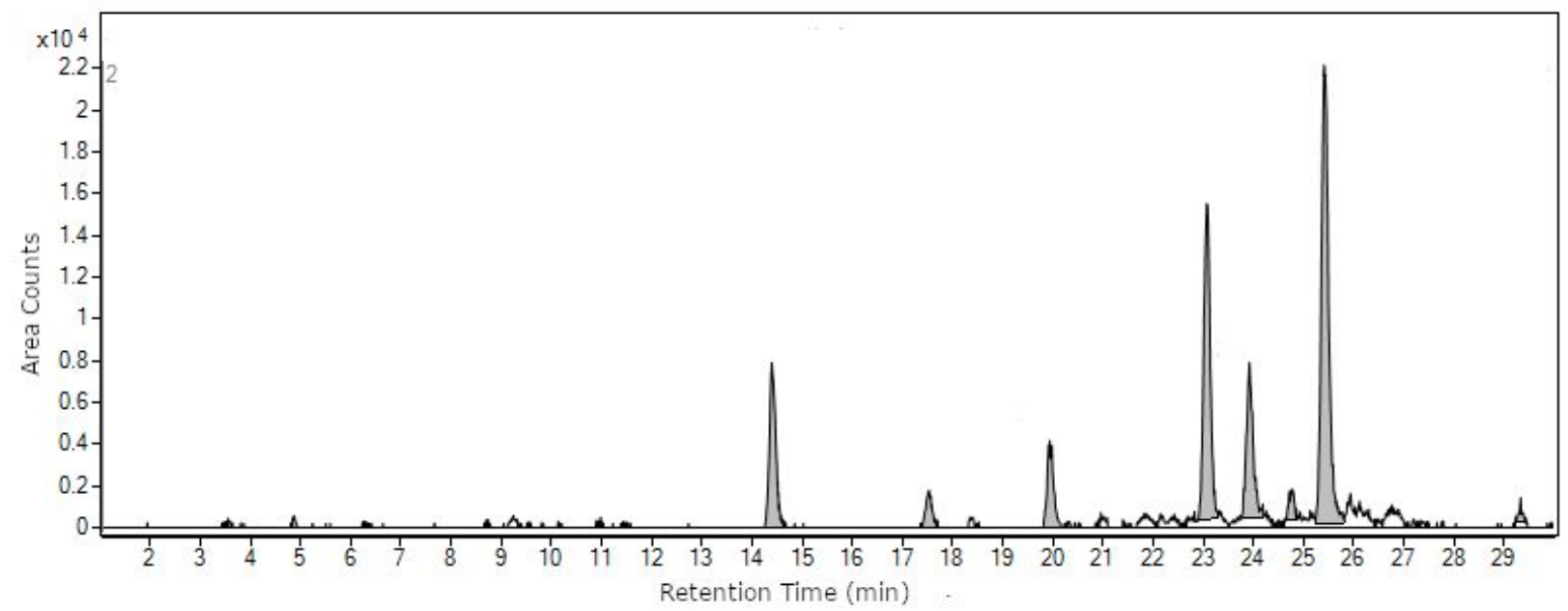

Figure S4. Extracted masses consistent with singly and doubly-derivatized hexanedione isomers extracted from a food waste digester biogas sample (bottom). Note that the retention times differ from those in the derivatized standard because the sample likely contains different structural isomers of hexanedione. 